\title{
Experimental studies to assess the potential of photodynamic therapy for the treatment of bronchial carcinomas
}

\author{
S G T Smith, J Bedwell, A J MacRobert, M H Griffiths, S G Bown, M R Hetzel
}

\begin{abstract}
Background-Photodynamic therapy (PDT) is a technique for producing localised tissue necrosis with light after prior administration of a photosensitising drug. There is some selectivity of uptake of photosensitisers in malignant tissue, although this is difficult to exploit. Full thickness necrosis in normal and neoplastic colon heals without perforation because of a lack of effect on collagen, making local cure a possibility. The experiments described here aim to establish whether these conclusions are also valid for bronchial tumours.
\end{abstract}

Methods-In pharmacokinetic studies normal rats were given $5 \mathrm{mg} / \mathrm{kg}$ of the photosensitiser aluminium sulphonated phthalocyanine (A1SPc) intravenously and killed up to one month later. The distribution of AISPc in the trachea was measured by chemical extraction and fluorescence microscopy. In subsequent experiments sensitised animals were treated with light delivered to the tracheal mucosa through a thin flexible fibre and the resultant lesions were studied for their size, mechanical strength, and healing. A series of resected human bronchial carcinomas were examined histologically for their collagen content. Results-The tracheal concentration of AlSPc in normal rats was maximum 1-20 hours after administration. Fluorescence microscopy revealed that most was in the perichondrium and submucosal stroma, with little in the cartilage. Light exposure showed necrosis of the soft tissues which healed by regeneration, but no effect on cartilage and no reduction in the mechanical strength of the trachea at any stage. Histological examination of resected human bronchial carcinomas showed more collagen in the tumour areas than would be found in normal regions.

Conclusions-PDT leads to necrosis of the soft tissues of the normal trachea but there is complete healing by regeneration, no risk of perforation (due to collagen preservation), and no effect on cartilage. Human bronchial carcinomas apparently contain more collagen than normal bronchi which may give protection against perforation following necro- sis induced by PDT. PDT may have a role in eradicating small volumes of tumour tissue in situ and could be valuable for treating (1) small carcinomas in patients unfit for resection, (2) tumour remaining after surgical resection, (3) stump recurrences, or (4) to prolong palliation of tumours after debulking with the NdYAG laser.

(Thorax 1993;48:474-480)

Only a minority of bronchial carcinomas are potentially curable by surgical resection and no real advances in cure rates are being achieved by radiotherapy or chemotherapy. New therapies therefore merit careful consideration. We have studied the potential role of photodynamic therapy (PDT) in the treatment of bronchial carcinoma and assessed its safety and efficacy in an animal model.

PDT is a technique for producing local tissue necrosis with light after prior administration of a photosensitising drug. The mechanism is photochemical rather than thermal, the active intermediate agent is considered to be singlet oxygen, ${ }^{1}$ so the intensity of light required is much lower than that used with high power instruments such as the neodymium yttrium aluminium garnet (NdYAG) laser. ${ }^{2}$ The technique has attracted considerable interest because photosensitisers are retained in malignant tissues with some selectivity compared with the adjacent normal tissues in which the tumour arose. The degree of selectivity, however, has been overemphasised in past publications and it is very difficult to limit damage to tumour areas when both tumour and normal tissue are exposed to the same light dose. ${ }^{3}$

Haematoporphyrin derivative $(\mathrm{HpD})$, or its partly purified form Photofrin (Quadralogic Technology, Vancouver, Canada), are the most commonly used photosensitisers for clinical PDT but they have certain disadvantages. Both are ill defined mixtures of porphyrins and leave patients sensitive to sunlight for several weeks after administration. Aluminium sulphonated phthalocyanine (AlSPc) is more promising ${ }^{4}$ and has been used experimentally in this centre since 1984 . It is relatively easy to synthesise, chemically 
stable, water soluble, and causes less photosensitivity to sunlight. ${ }^{5}$ Red light is used for PDT as it penetrates tissue much better than other visible wavelengths but is still able to activate the photosensitisers. AlSPc has the additional advantage that it absorbs red light much better than HpD. Following the finding that AlSPc enabled the photodestruction of sensitised cells in vitro, ${ }^{6}$ the uptake, distribution and biological effects of this sensitiser were investigated in vivo in a number of normal and malignant tissues. ${ }^{7-11}$ AlSPc has proved to be a more efficient sensitiser than $\mathrm{HpD}$ although its selectivity of tumour uptake is the same. ${ }^{7}$

There have been few reports of experiments with PDT on bronchial tumours in animals, ${ }^{12}$ and none on its effects on normal bronchi although detailed studies have been carried out in the gastrointestinal and urinary tracts. ${ }^{313-15}$ Most of the data on uptake of photosensitisers by bronchial tumours are clinical and based on fluorescence excited by ultraviolet (UV) light and visualised by bronchoscopy. ${ }^{16}$ UV light only penetrates a fraction of a millimetre into tissue so the technique only looks at surface changes, but as far as can be judged from these data, the selectivity of uptake of the sensitisers in bronchial tumours is likely to be comparable to that in colonic tumours. If there is to be any prospect of eradicating small bronchial tumours by PDT it is therefore essential to understand the effects of PDT on normal bronchi.

The main conclusions from the experimental work carried out on the rat colon were that necrosis produced by PDT healed safely in normal and neoplastic areas without any reduction in the mechanical strength of the bowel wall because of a lack of any effect of PDT on collagen. The aim of the present work was to establish whether similar conclusions could be drawn about the effect of PDT on normal airways, and to look at the collagen content of bronchial cancers to assess whether treatment of these tumours with PDT is likely to cause any weakness in the bronchial wall. Experiments were carried out on the trachea of normal rats to study the distribution of the photosensitiser and the response to light. Lesions produced by PDT were compared with those produced thermally. In addition the collagen content of non-small cell human lung cancers was examined in resected specimens.

\section{Methods}

IN VIVO STUDIES ON THE NORMAL RAT TRACHEA

The distribution of photosensitiser in the normal rat trachea at a range of times after administration was studied by a chemical extraction technique and fluorescence microscopy. The effects of PDT were produced by exposing the trachea of sensitised animals to red light. Lesions of similar size were produced by low power hyperthermia in unsensitised animals and the mechanical strengths of the two types of lesion were compared with the strength of normal trachea. Similar lesions (PDT only) were produced in a separate series of experiments for assessment of healing.

Photosensitiser and animals

AlSPc (Ciba-Geigy) was dissolved in normal saline at a concentration of $2 \mathrm{mg} / \mathrm{ml}$ and the solution was stored in the dark. All in vivo experiments were performed on adult male Wistar rats (400-800 g) sensitised with a 5 $\mathrm{mg} / \mathrm{kg}$ intravenous dose of AlSPc. All injections and surgical procedures were performed under general anaesthesia induced by intramuscular Hypnorm (fentanyl and fluanisone).

\section{Assay of AlSPc by chemical extraction}

Animals were killed at times from five minutes to one month after sensitisation and the relevant tissues removed and stored at $-4^{\circ} \mathrm{C}$ until required for assay. AlSPc levels were determined in both lung and trachea. At least three animals were used for each data point.

Thawed tissues were assayed with an alkaline extraction technique. ${ }^{17}$ Briefly, wet tissue was treated with $0.1 \mathrm{~mol} / 1 \mathrm{NaOH}$ in a ratio of $0.1 \mathrm{~g}: 10 \mathrm{ml}$. Samples were then placed in a water bath at $50^{\circ} \mathrm{C}$ for four hours with regular agitation and AlSPc concentrations in the supernatant were determined with a fluorometric assay by comparison of fluorescence with that from a standard curve produced using unsensitised tissue and known quantities of sensitiser. The spectrofluorimeter (Perkin-Elmer LS-5 Luminescence Spectrofluorimeter) was set to excite at $604 \mathrm{~nm}$ and to detect at $673 \mathrm{~nm}$ (10 nm slit widths) with a filter to remove scattered light below $645 \mathrm{~nm}$. Results were expressed as $\mu \mathrm{g} / \mathrm{g}$ wet tissue.

\section{Microscopic distribution of AlSPc}

Animals were killed at times from five minutes to four hours after sensitisation and the trachea removed and immediately snap frozen. To preserve cellular integrity samples were frozen with isopentane cooled in liquid nitrogen. Serial sections were then prepared, one section being kept frozen while the adjacent section was fixed and stained with haematoxylin and eosin. Cellular detail could thus be observed under a light microscope with the haematoxylin and eosin sections and correlated with the fluorescence of the unstained sections seen with a charged coupled device imaging system (Wright Instruments, Cambridge). The charged coupled device allowed quantitative fluorescence imaging of AlSPc in tissues. Fluorescence was excited with an $8 \mathrm{~mW}$ helium neon laser operating at $632.8 \mathrm{~nm}$ and detected in the range $660-700 \mathrm{~nm}$ as previously described. ${ }^{11}$ Control sections were examined to quantify and correct for autofluorescence from unsensitised tissues.

\section{Photodynamic therapy}

Photodynamic therapy of the normal rat trachea was carried out under general anaes- 
Figure 1 Mean (SD) concentration of AlSPC extracted from normal lung and trachea over time after a $5 \mathrm{mg} / \mathrm{kg}$ dose $(n=3-8$ per point).

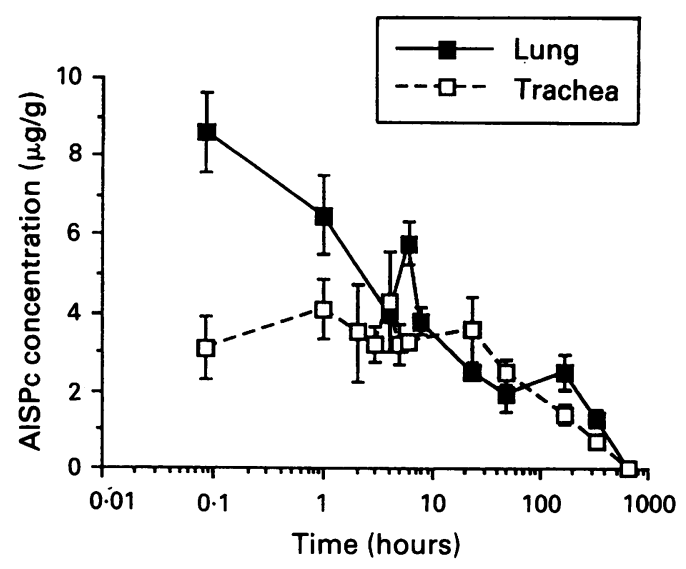

thesia as an open procedure one hour after intravenous administration of $5 \mathrm{mg} / \mathrm{kg}$ AlSPc. The trachea was exposed by a midline neck incision and a $200 \mu \mathrm{m}$ laser fibre was introduced by a small stab wound so that the

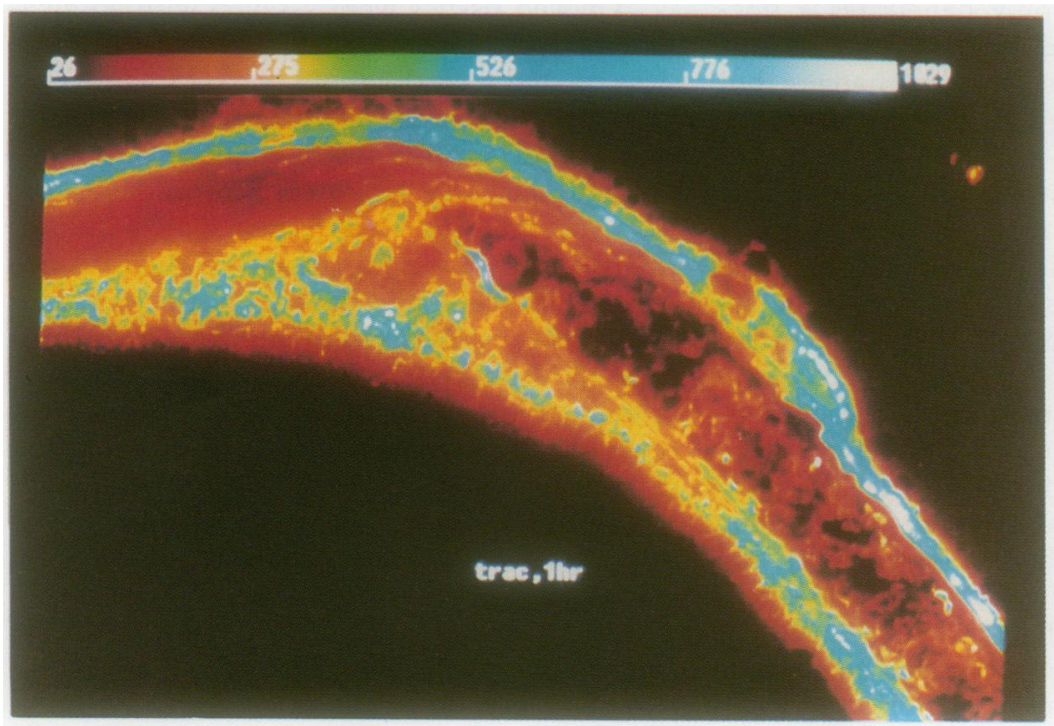

(A)

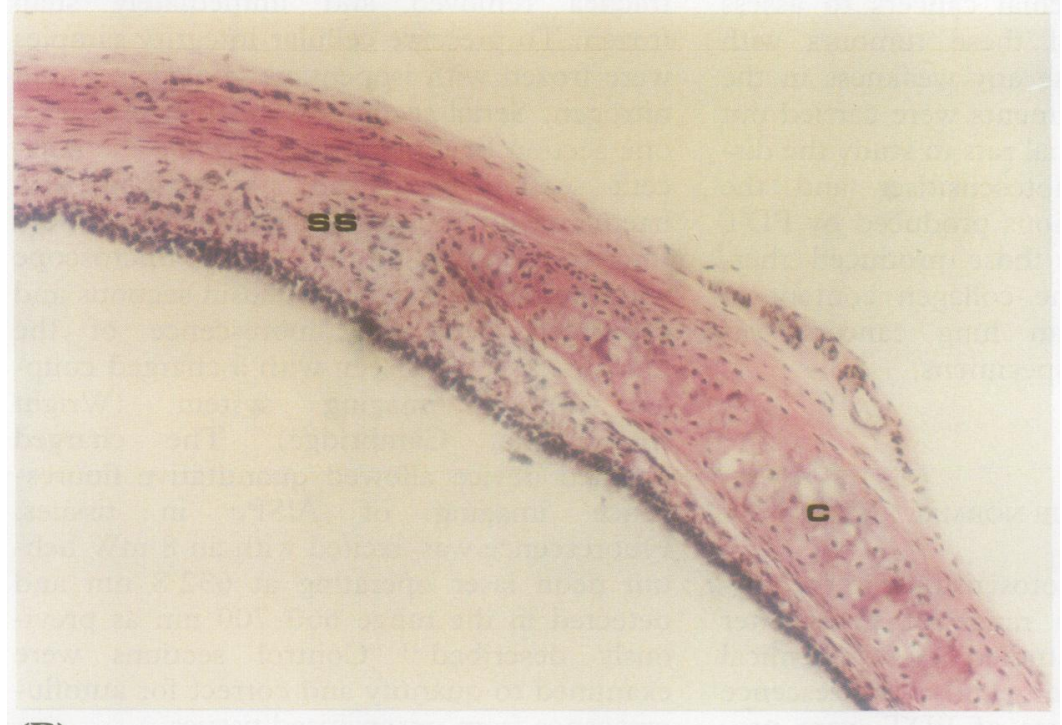

(B)

Figure 2 (A) Fluorescence microscopy of trachea one hour after administration of $5 \mathrm{mg} / \mathrm{kg}$ AlSPc intravenously. Intensity is shown on the false colour code with scale at the top (low levels: black and red, high levels: blue and white). (B) Adjacent section to (A) stained with haematoxylin and eosin. ss-submucosal stroma, $c$-cartilage.

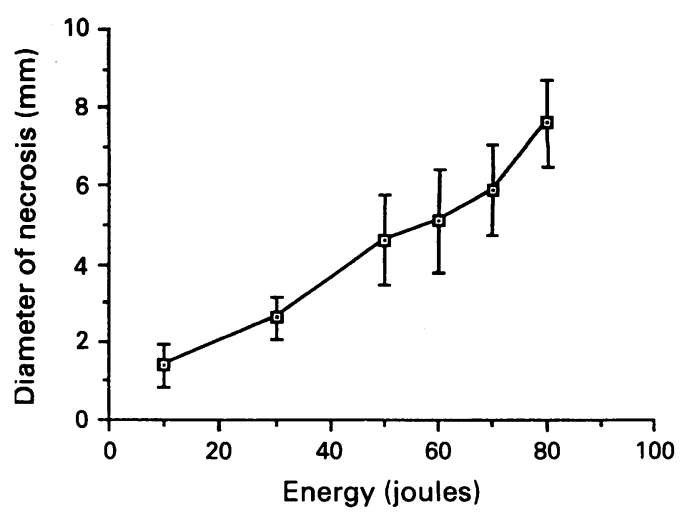

Figure 3 Mean (SD) diameter of PDT damage to the trachea of five animals as a function of the applied energy. All treatments were at one hour after sensitisation with

$5 \mathrm{mg} / \mathrm{kg}$ using a power of $100 \mathrm{~mW}$.

tip just touched the mucosa on the opposite side of the trachea. Red light (675 $\mathrm{nm}$ ) was produced by a copper vapour pumped dye laser (Oxford Lasers, Oxford) and total energies of $10-100 \mathrm{~J}$ (100 mW for $100-1000 \mathrm{sec}-$ onds) were applied (one lesion per animal). The laser power used was $100 \mathrm{~mW}$ as preliminary experiments had shown that this was the highest power that could be used without causing charring at the fibre tip. The surgical wound was repaired and the animal allowed to recover. Three aspects of the response to PDT were studied: (1) to correlate the size of the lesion with the applied energy, energies from 10-100 J were used (five animals for each energy), animals were killed 72 hours after light exposure, and the diameter of the PDT lesion was measured macroscopically on the fresh specimen; (2) for histological examination ( $100 \mathrm{~J}$ lesions only) animals were killed at times from two hours to three months after light exposure, the trachea was removed and opened, the PDT lesion identified, and the specimen trimmed and fixed in formalin; (3) to test tracheal compliance and bursting pressure 24 hours after light exposure (described below) the mechanical

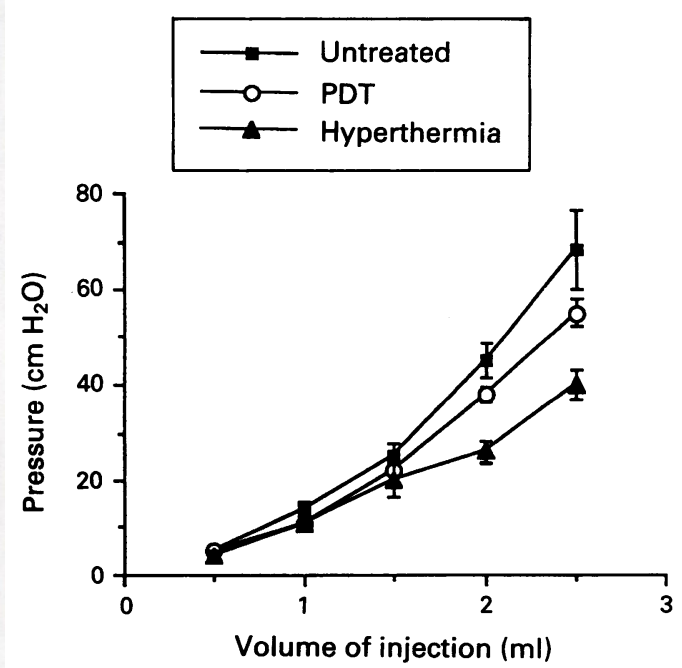

Figure 4 Mean (SD) tracheal compliance in untreated animals and after PDT (80 F one hour after sensitisation) and low power hyperthermia (100 $\mathcal{f}$ at 1 W). All measurements recorded 24 hours after treatment $(n=10)$. 


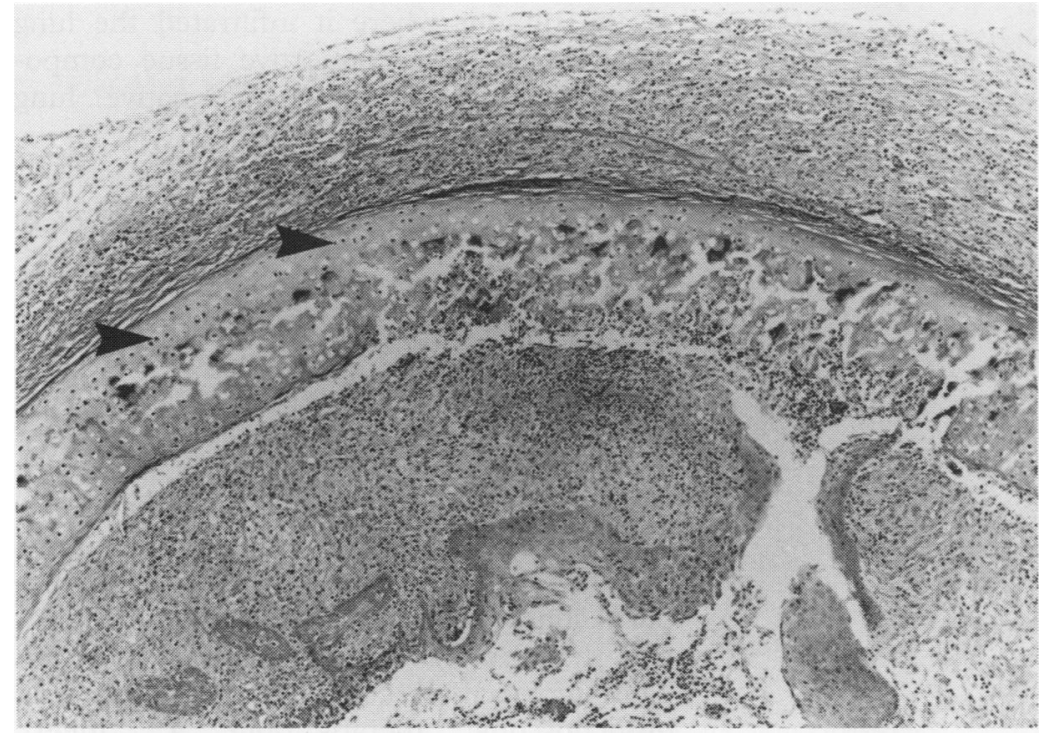

Figure 5 Section of trachea two weeks after treatment with PDT showing necrosis and inflammation in the soft tissue on either side of the tracheal cartilage but retention of the chondrocyte nuclei (arrows) indicating that the cartilage has remained viable. The trachea is lined by regenerated epithelium of squamous type (van Gieson stain).

strength of PDT and thermal lesions was compared with untreated controls, thermal lesions of similar size being created in unsensitised animals by a NdYAG laser at a power of $1 \mathrm{~W}$ to deliver an energy of $100 \mathrm{~J}(100 \mathrm{sec}-$ ond exposure time) and tested after 24 hours, two weeks, and two months. Ten animals were used in each group (three thermal groups, one PDT and one control).

\section{Compliance and bursting pressure of trachea}

Tracheal compliance and bursting pressure were measured with a purpose built instrument. ${ }^{8}$ Each excised trachea treated by PDT or hyperthermia, or untreated control, was clamped at both ends (one end around a cannula) and filled with water in $0.5 \mathrm{ml}$ aliquots up to the bursting point. Pressure measurements were recorded after each addition of water with a fluid filled line $(8 \mathrm{G})$ connected to a transducer which was calibrated against a manometer giving the pressure in $\mathrm{cm} \mathrm{H}_{2} \mathrm{O}$. The bursting pressure was taken as the pressure in the trachea just before rupture indicated by leakage of water. The trachea was examined after bursting and the site of rupture in relation to the laser lesion noted.

\section{HISTOLOGY OF HUMAN BRONCHIAL CANCERS}

In a separate study eight resected human cancers of major bronchi received consecutively in the department of histopathology were examined to assess their collagen content. Sections were chosen to show the region where the tumour had destroyed the normal bronchial wall and were stained by the van Gieson method to show their collagen content.

\section{Results}

IN VIVO STUDIES ON NORMAL RAT TRACHEA Assay of AlSPc by chemical extraction

The concentration of AlSPc in trachea and lung from five minutes to one month after
Mean (SD) tracheal bursting pressure of untreated, PDT treated ( $80 \mathrm{f}$ one hour after sensitisation) and low power hyperthermia treated $(100$ f) samples $(n=10)$.

\begin{tabular}{ll}
\hline Tracheal protocol & $\begin{array}{l}\text { Bursting pressure } \\
\left(\mathrm{cm} \mathrm{H}_{2} \mathrm{O}\right)\end{array}$ \\
\hline Untreated trachea & $98(6)$ \\
PDT (24 hours) & $95(8)$ \\
Hyperthermia (24 hours) & $48(6)$ \\
Hyperthermia (2 weeks) & $59(8)$ \\
Hyperthermia (2 months) & $73(7)$ \\
\hline
\end{tabular}

injection of a $5 \mathrm{mg} / \mathrm{kg}$ dose is shown in fig 1 . The lung shows an initial high level of AlSPc at five minutes after injection which is not present with the trachea, probably because of high blood levels in the lung parenchyma. The maximum uptake of sensitiser in the trachea was seen 1-20 hours after administration with a subsequent decrease in concentration, none being detectable after a month. One hour was chosen as a convenient time interval for the subsequent experiments on PDT.

\section{Microscopic distribution of AlSPc}

Fluorescence microscopy examinations of tracheal sections revealed a distinct highly fluorescent area surrounding the cartilage which corresponded to the perichondrium. This reflects the vascular nature of this membrane. In all samples studied (five minutes, one and four hours after sensitisation) this was the area of greatest fluorescence together with the submucosal stroma. There was little or no fluorescence in other areas, particularly the cartilage, which showed very low uptake of AlSPc. The fluorescence pattern one hour after sensitisation is shown in fig $2 \mathrm{~A}$ together with the adjacent section from the same block stained with haematoxylin and eosin (fig 2B).

\section{Photodynamic therapy}

The diameters of the necrotic zones produced in the trachea by energies of 10-80 J are shown in fig 3. All animals were killed 72 hours after exposure to light. The lesion size increased with the delivered energy up to $80 \mathrm{~J}$, the maximum that the animals could tolerate. One animal given $100 \mathrm{~J}$ died from oedema at the treatment site which caused tracheal obstruction.

\section{Compliance and bursting pressure of trachea}

The results are shown in fig 4 . The tracheal compliance 24 hours after PDT with $80 \mathrm{~J}$, one hour after sensitisation, was only slightly reduced from that of the untreated tissue although a greater reduction was seen 24 hours after low power hyperthermia (100 J). The greater damage to the structural integrity of normal trachea by hyperthermia is particularly obvious from the bursting pressures shown in the table. At no time after PDT was a significant reduction in bursting pressure observed when compared with untreated tracheas. The specimens treated with PDT exhibited no specific site of bursting. In contrast, the bursting pressure 24 hours after 


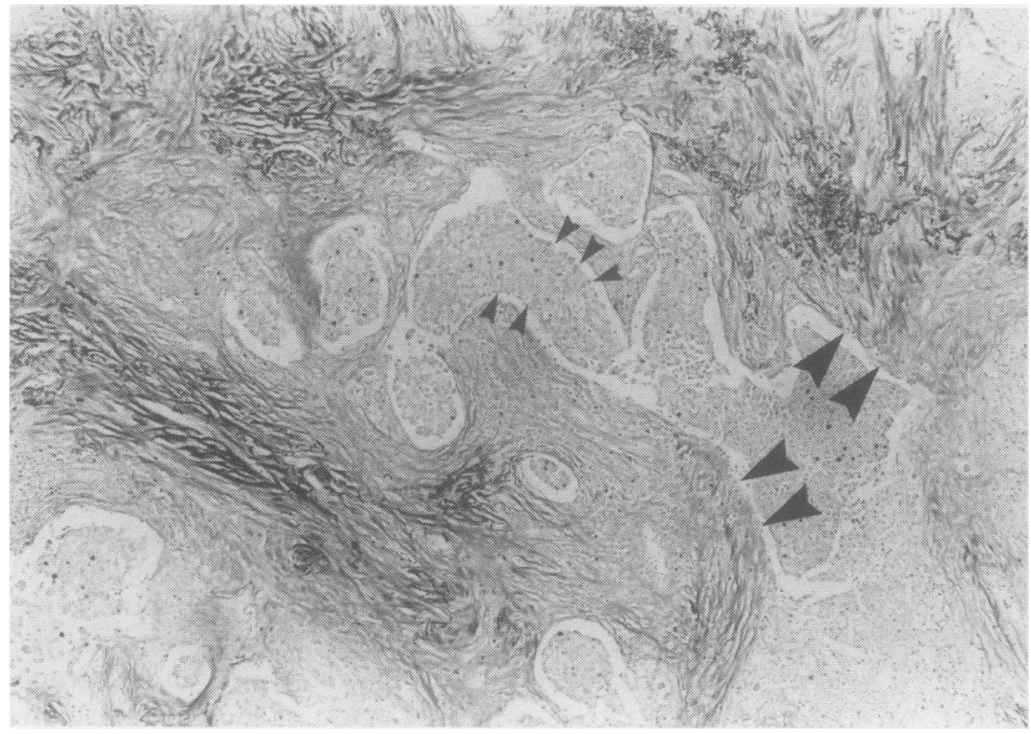

Figure 6 Section of resected human bronchial squamous cell carcinoma showing the relative predominance of collagenous stroma (large arrows) over the paler staining islands of tumour (small arrows) in the central (bronchial) parts of the tumour. The stroma occupies approximately $75 \%$ of the area of the section (van Gieson stain).

hyperthermia was reduced by about $50 \%$ compared with that of untreated tissue, and all these samples burst through the lesion. This reduction in bursting pressure did not return to normal even after two months (table).

\section{Histology of PDT lesions}

No change was seen in sections of trachea two hours after PDT. At 24 hours the mucosa appeared oedematous and inflammatory exudate was observed in the lumen. At 72 hours there was focal epithelial and subepithelial necrosis with associated local acute inflammation. At one week patchy necrosis was present in the mucosa, the submucosa, and the soft tissues outside the tracheal cartilage, but the cartilage appeared viable. At two weeks (fig 5) there was circumferential thickening of the mucosa by inflamed granulation tissue lined partly by regenerated (squamous) epithelium and partly by ciliated columnar epithelium. Granulation tissue was also present outside the cartilage which remained intact. The mucosa distal to this stenosing mucosal thickening was hyperplastic and acute inflammatory exudate was noted in the lumen. By two months the histology of the trachea had returned to normal and remained so at three months.

\section{HISTOLOGY OF RESECTED HUMAN BRONCHIAL} CARCINOMAS

The relative proportions of tumour to collagenous stroma per low power field were estimated in sections from eight bronchial non-small cell carcinomas (six squamous, one large cell, and one adenocarcinoma) after staining by the van Gieson method. In all tumours the proportion of collagen to tumour was very high $(30-75 \%)$ in the region of the bronchus - the presumed origin of the tumour-but fell away towards the periphery of the tumour where it infiltrated the lung parenchyma. The connective tissue component here was that of the native lung parenchyma (fig 6).

\section{Discussion}

Most early reports of the use of PDT for treating bronchial cancers focused on the commonest clinical problem-that is, palliation of advanced, inoperable tumours. It was proposed that PDT should be used for the removal of exophytic tumour causing obstruction of major airways. These early treatments were carried out on an empirical basis with few experimental data on which to base the techniques and treatment methods used, and the results were very disappointing. ${ }^{18-20}$ Tumour necrosis could be produced with bronchoscopic therapy but a "toilet" bronchoscopy was required a few days after the initial treatment to remove the dead tissue and associated secretions. More seriously, oedema in the treated tumour could convert partial bronchial obstruction to complete obstruction which could be life threatening if there was no adequately ventilated lung tissue elsewhere. In addition, in one of these early reports two of six patients with advanced cancers died of massive secondary haemorrhage six and 11 days after treatment, ${ }^{18}$ and most subsequent reports of treatment of advanced tumours have included similar mortalities. There is unlikely to be any role for PDT as the primary treatment for advanced bronchial cancers; endoscopic recanalisation of bronchi obstructed by exophytic tumour is carried out much more safely and effectively with the NdYAG laser. Palliative recanalisation can usually be completed in one treatment under general anaesthesia without the need for a "clean up" bronchoscopy or the problems of delayed haemorrhage or sensitisation of skin to sunlight. $^{2}$

With small tumours, however, the situation is completely different. In the same study that reported fatal haemorrhage after PDT in two advanced cancers there was a complete response to treatment (no tumour visible at follow up bronchoscopy) with no complications in five cases of early cancer. ${ }^{18}$ Other groups have obtained similar results with early cancers not recurring at up to three years. ${ }^{21} 22$

It is easy to destroy a small volume of almost any solid tumour by PDT. ${ }^{23}$ What matters to a patient is whether that tumour can be destroyed safely and completely in the tissue where it arises. The effect of treatment and subsequent healing on immediately adjacent normal tissue must be understood. The purpose of this paper is to understand what PDT does to normal bronchial tissue and to suggest the maximum diameter of bronchial tumour which might be suitable for PDT. Such studies have not been reported previously.

The results show that full thickness necrosis produced by PDT in the rat trachea 
heals safely with no cartilage damage or reduction in bursting pressure, so it is unlikely that PDT will cause perforation or collapse of normal major airways. In contrast, thermal lesions damage cartilage and reduce the strength of the trachea. It is therefore potentially dangerous to treat normal tissues with the NdYAG laser, which makes the two laser techniques complementary. The NdYAG is better for palliative debulking of large tumours, while PDT is better for ablating small cancers in their entirety when some normal areas will inevitably be exposed to treatment. Care must be taken, however, to ensure that the power of the laser used for PDT is not sufficient to cause thermal effects such as probably occurred in the development of tracheo-oesophageal fistulae after PDT to oesophageal tumours, a complication that the experimental data suggest should not be experienced after PDT. ${ }^{24}$

The purpose of the present experiments was to understand the nature of damage to the airways caused by PDT rather than to optimise dosimetry, so the experiments were carried out at a time when the tissue concentration of sensitiser was at its highest (one hour after administration). Most studies in solid tumours suggest that the maximum ratio of tumour to normal tissue concentration of sensitiser occurs 24-48 hours after sensitisation which is why this time interval is often used clinically, although the tumour: normal ratio is seldom more than $3: 1,{ }^{14} 25-27$ and it is extremely difficult to exploit this ratio to achieve truly selective tumour necrosis. Better selectivity with a shorter time interval and less skin sensitivity may be possible with the new sensitiser precursor, 5amino laevulinic acid (ALA) ${ }^{28}$

The possibility of delayed secondary haemorrhage resulting from PDT of advanced bronchial cancers is extremely worrying. ${ }^{18}$ 20 This has been addressed in colonic cancers of rats treated with PDT; three of nine tumours over $2 \mathrm{~cm}$ in diameter, but none of 24 smaller lesions, showed evidence of delayed haemorrhage. ${ }^{3}$ The main feature of the lesions that bled was that they had been substantially undertreated (margin between necrotic and viable areas lying in tumour tissue), confirming that it is wiser to limit PDT to small lesions.

Most malignant bronchial tumours destroy at least some of the normal tissues in which they arise. It is of no value to destroy a cancer if the result is a perforated bronchus due to full thickness destruction of the bronchial wall by the original tumour. PDT can only be of value clinically for possible tumour eradication if part of the normal bronchial wall remains or there is some connecting tissue within the tumour that is sufficiently resistant to PDT to preserve the mechanical integrity of the airway at all stages of healing. In studies on the rat colon collagen has been shown to perform this role in normal and neoplastic areas. ${ }^{315}$ Similar results were found on resected human colonic cancers. ${ }^{3}$ This is important as it implies that small, but full thickness, tumours of the bowel, together with a margin of adjacent normal tissue, can be treated with PDT with little risk of immediate or delayed perforation. It is difficult to be sure that the collagen in a cancer will be well enough organised to provide the same mechanical strength as that in normal tissue, but the results from the treated rat colonic cancers suggest that it is sufficient. ${ }^{3}$ The histological studies on human bronchial cancers reported here show similar amounts of collagen to the human colonic cancers $^{3}$ which suggests that it may be safe to use PDT to treat small bronchial cancers that involve the full thickness of the bronchial wall, together with a margin of normal tissue, without risk of perforation. Early cancers that do not involve the full thickness of the bronchial wall present an intermediate situation. They probably contain less collagen than the advanced ones but, on the other hand, they still have the strength of the remaining normal parts of the bronchial wall. No appropriate histological data are available on such lesions. It is important to know whether they could be more vulnerable to perforation than either normal areas or full thickness lesions, although this seems unlikely as it is rare for tumour containing areas of bronchus to be thinner than adjacent normal regions.

Treatment of full thickness lesions with PDT will require extreme care. The size of the tumour will always be a limiting factor because of the risk of secondary haemorrhage but, even for relatively small tumours, spontaneous breaches of the bronchial wall may occur such as the development of tracheo-oesophageal fistulae, so there is nothing "absolute" about the collagen bridge across the tumour and the risk would have to be assessed carefully in every case.

These results make it feasible to speculate on how PDT may be clinically useful. The most likely situation is with a small volume of tumour of definable extent in a patient unsuitable for surgery. PDT after surgery might kill any tumour cells left in the resection margin and so prevent stump recurrence or it could be used to treat stump recurrences. Another possibility would be to treat the base of exophytic tumours debulked with the NdYAG laser. This could prolong the relatively short period of remission of symptoms achieved with the NdYAG alone. One study reported that PDT applied before surgery may reduce the extent of resection required so that an otherwise inoperable patient may become operable, although this might lead to the problems mentioned above associated with partial necrosis of cancers. ${ }^{21}$ Like all laser therapy PDT is a local treatment and, although the photosensitiser is distributed widely, there will only be a biological effect where there is also therapeutic light. For there to be any prospect of cure it is essential to know how far the tumour extends, both in depth and in local spread along the bronchial lumen, and to be able to deliver an adequate light dose to all relevant 
areas both on and below the mucosal surface. These are major challenges in themselves, particularly for thicker lesions as the red light used penetrates only a few millimetres into tissue but, as normal bronchial tissue can tolerate PDT so well, normal tissue beyond the likely margins of the tumour can be treated safely which reduces the accuracy of tumour mapping required before potentially curative treatment.

Surgery currently offers the only chance of cure for carcinoma of the bronchus but only about $10 \%$ of patients are fit for surgery. The overall five year survival for all lung cancers, allowing for recurrences after surgery, is about $8 \%$. Any technique such as PDT which might be capable of replacing surgery or reducing the extent of surgery necessarily merits serious consideration in a disease which currently causes 30000 deaths each year in England and Wales, although the potential for cure will be limited to the relatively small percentage of tumours that are still localised to the region of origin at the time of treatment.

This work was funded by the Special Medical Development on Lasers from the Department of Health and by the Imperial Cancer Research Fund. Dr MacRobert was also supported by the Waldburg Trust.

During the preparation of this manuscript, Mr Simon Smith, FRCS, research fellow, was tragically killed in a road traffic accident.

Weishaupt K, Gomer CJ, Dougherty TJ. Identification of singlet oxygen as the cytotoxic agent in photo-radiation of a murine tumour. Cancer Res 1976;36:2326-9.

2 Hetzel MR, Smith SGT. Endoscopic palliation of tracheobronchial malignancies. Thorax 1991;46:325-33.

3 Barr H, Chatlani P, Tralau CJ, MacRobert AJ, Boulos PB, Bown SG. Local eradication of rat colon cancer with photodynamic therapy: correlation of distribution of photosensitiser with biological effects in normal and tumour tissue. GUT 1991;32:517-23.

4 Spikes JD. Phthalocyanines as photosensitisers in biological systems and for the photodynamic therapy of tumours. Photochem Photobiol 1986;43:691-9.

5 Tralau CJ, Young AR, Walker NPJ, Vernon DI, MacRobert AJ, Bown SB, et al. Mouse skin photoMacRobert AJ, Bown SB, et al. Mouse skin photosensitivity with dihaematoporphyrin ether (DHE) and
aluminium sulphonated phthalocyanine (AlSPc): a comparative study. Photochem Photobiol 1989;49: 305-12.

6 Chan WS, Svensen R, Phillips D, Hart IR. Cell uptake, distribution and response to aluminiúm chloro phthalocyanine, a potential anti-tumour photosensitiser. $\mathrm{Br} \mathfrak{F}$ Cancer 1986;53:255-64.

7 Tralau CJ, Barr H, Sandeman DR, Barton T, Lewin MR, Bown SG. Aluminium sulfonated phthalocyanine distribution in rodent tumors of the colon, brain and pancreas. Photochem Photobiol 1987;46:777-81.

8 Barr H, Tralau CJ, MacRobert AJ, Krasner N, Boulos PB Clark CG, et al. Photodynamic therapy in the normal rat colon with phthalocyanine sensitisation. $\mathrm{Br} f \mathrm{Cancer}$ 1987;56:111-8.
9 Bown SG, Tralau CJ, Coleridge-Smith PD, Akdermir D. Wieman TJ. Photodynamic therapy with porphyrin and phthalocyanine sensitisation: quantitative studies in normal rat liver. $B r \mathcal{F}$ Cancer 1986;54:43-52.

10 Tralau CJ, MacRobert AJ, Coleridge-Smith PD, Barr H, Bown SG. Photodynamic therapy with phthalocyanine sensitisation: quantitative studies in a transplantable rat fibrosarcoma. $B r f$ Cancer 1987;55:389-95.

11 Pope AJ, MacRobert AJ, Phillips D, Bown SG. The detection of phthalocyanine fluorescence in normal rat bladder wall using sensitive digital imaging microscopy. $\mathrm{Br} F$ Cancer 1991;64:875-9.

12 Hayata $\mathrm{Y}$, Kato $\mathrm{H}$, Konaka C, Hayashi N, Tahara M, Saito T. Fiberoptic bronchoscopic photoradiation in experimentally induced canine lung cancer. Cancer 1983 51:50-6.

13 Pope AJ, Bown SG. The morphological and functional changes in rat bladder following photodynamic therapy with phthalocyanine photosensitisation. F Urol 1991; 145:1064-70.

14 Barr H, Tralau CJ, Boulos PB, MacRobert AJ, Krasner N, Phillips $\mathrm{D}$, et al. Selective necrosis in DMH induced rat colon tumours using phthalocyanine photodynamic therapy. Gastroenterology 1990;98:1532-7.

15 Barr H, Tralau CJ, Boulos PB, MacRobert AJ, Tilly R, Bown SG. The contrasting mechanisms of colonic collagen damage between photodynamic therapy and thermal injury. Photochem Photobiol 1987;46:795-800.

16 Hayata Y, Kato H, Konaka C, Ono J, Matsushima Y, Yoneyama K. Fiberoptic bronchoscopic laser photoradiation for tumor localization in lung cancer. Chest 1982;82:10-4.

17 Chan WS, Marshall JF, Lam GYF, Hart IR. Tissue uptake, distribution and potency of the photoactivatable dye chloroaluminium sulfonated phthalocyanine in mice
bearing transplantable tumors. Cancer Res 1988;48: bearing

18 Cortese DA, Kinsey JM. Endoscopic management of lung cancer with HpD. Mayo Clin Proc 1982;57: 543-7.

19 Vincent RG, Dougherty TJ, Rao U, Boyle DG, Potter W. Photoradiation therapy in the treatment of advanced carcinoma of the trachea and bronchus. In: Doirin DR, Gomer CG, eds. Porphyrin localization and treatment of tumors. New York: Alan Liss, 1984.

20 Balchum OJ, Doirin DR. Photoradiation therapy of obstructing endobronchial lung cancer, employing the photodynamic action of $\mathrm{HpD}$. In: Doirin DR, Gomer
CG, eds. Porphyrin localization and treatment of tumors. CG, eds. Porphyrin localization

21 Hayata Y, Kato H, Konaka C, Amemiya R, Ono J, Ogawa $\mathrm{I}$, et al. Photoradiation therapy with $\mathrm{HpD}$ in early and stage 1 lung cancer. Chest 1984;86:169-77.

22 Konaka $C$, Kato $H$, Hayata $Y$. Lung cancer treated by photodynamic therapy alone: survival for more than three years. Lasers Med Sci 1987;2:17-9.

23 Henderson BW, Waldow SM, Mang TS, Potter WR, Malone PB, Dougherty TJ. Tumor destruction and kinetics of tumor cell death in two experimental mouse tumors following PDT. Cancer Res 1985;45:572-6.

24 Thomas RJ Abbott $M$, Bhathal PS, St John DJB, Morstyn G. High dose photoirradiation of oesophageal cancer. Ann Surg 1987;206:193-9.

25 Bown SG. Photodynamic therapy to scientists and clinicians: one world or two? $\mathcal{f}$ Photochem Photobiol, B Biology 1990;6:1-12.

26 Agrez MV, Wharen RE, Anderson RE, Laws ER, Ilstrup DM. HpD quantitative uptake in $\mathrm{DMH}$ induced murine colorectal carcinoma. $\mathcal{F}$ Surg Oncol 1983;24: murine

27 Chan WS, Marshall JF, Hart IR. Effect of tumour location on selective uptake and retention of phthalocyanines. Cancer Lett 1989;44:73-7.

28 Bedwell J, MacRobert AJ, Phillips D, Bown SG. Fluorescence distribution and photodynamic effect of ALA-induced PP IX in the DMH rat colon tumour model. Br f Cancer 1992;65:818-24. 